\title{
The Dynamics of Active Metal Catalysts Revealed by In Situ Electron Microscopy
}

\author{
Zhu-Jun Wang, Ramzi Farra, Jing Cao, Robert Schlögl and Marc Georg Willinger
}

Department of Inorganic Chemistry, Fritz-Haber-Institut der Max-Planck-Gesellschaft, Berlin, Germany.

Conventional high-resolution imaging by electron microscopy plays an important role in the structural and compositional analysis of catalysts. However, since the observations are generally performed under vacuum and close to room temperature, the obtained atomistic details concern an equilibrium state that is of limited value when the active state of a catalyst is in the focus of the investigation. Since the early attempts of Ruska in 1942 [1], in situ microscopy has demonstrated its potential and, with the recent availability of commercial tools and instruments, led to a shift of the focus from ultimate spatial resolution towards observation of relevant dynamics [2].

During the last couple of years we have implemented commercially available sample holders for in situ studies of catalysts in their reactive state inside a transmission electron microscope. In order to relate local processes that occur on the nanometer scale with collective processes that involve fast movement of a large amount of atoms, we have adapted an environmental scanning electron microscope (ESEM) for the investigation of surface dynamics on active catalysts. Using these two instruments, we are now able to cover a pressure range from $10^{-4}$ to $10^{3} \mathrm{mbar}$ and a spatial resolution ranging from the $\mathrm{mm}$ to the sub-nm scale.

Presently we are investigating the behavior of metal catalysts during hydrocarbon partial oxidation and decomposition reactions as well as structural dynamics during oscillatory red-ox reactions. The observations are performed in real-time and under conditions in which the active state of the catalyst can be monitored. The latter is of upmost importance, since the key requirement is to observe relevant processes and dynamics that are related to catalytic function.

The ability to directly image the active catalyst and associated morphological changes at high spatial resolution enables us to refine the interpretation of spatially averaged spectroscopic data that was obtained under otherwise similar reaction conditions, for example during near-ambient-pressure in situ XPS measurements [3].

In the case of metal catalyzed chemical vapor deposition growth of graphene, we are able to observe nucleation and growth of graphene domains in real time and to study the effect of catalyst surface termination, atmosphere and temperature in a very flexible way (Figure 1) [4].

It will be shown that the ability of observing the adaption of an active surface to changes in the chemical potential of the surrounding gas phase in real-time potentially offers new and direct ways of optimizing catalysts and applied reaction conditions. One of the examples that will be demonstrated is the dynamic state of copper, where the simultaneous and very dynamic formation of oxidized and reduced islands is observed during red-ox conditions in different atmospheres and on different length scales (Figure 2). 


\section{References:}

[1] E Ruska, Kolloid-Zeitschrift 100 (1942), pp. 212-219.

[2] SB Vendelbo et al, Nat. Mater. 13 (2014), pp. 884-890.

[3] R Blume et al, PhysChemChemPhys 16 (2014), p. 25989.

[4] ZJ Wang et al, ACS Nano 9 (2015), pp. 1506-1519.
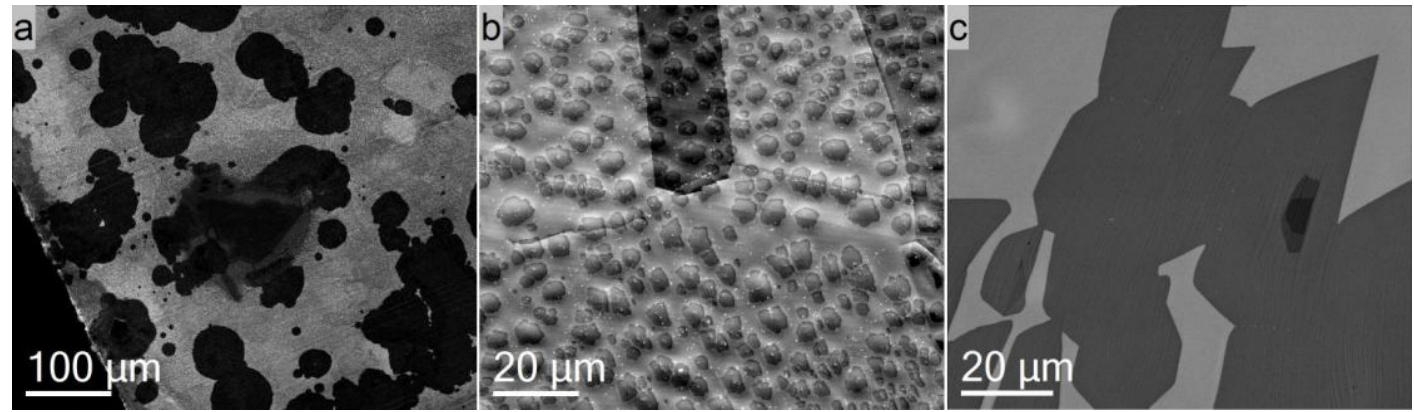

Figure 1. Snapshots taken during metal catalyzed CVD growth of graphene in the chamber of the ESEM for the case of $\mathrm{Ni}$ (a), $\mathrm{Cu}$ (b) and $\mathrm{Pt}$ (c) substrates.
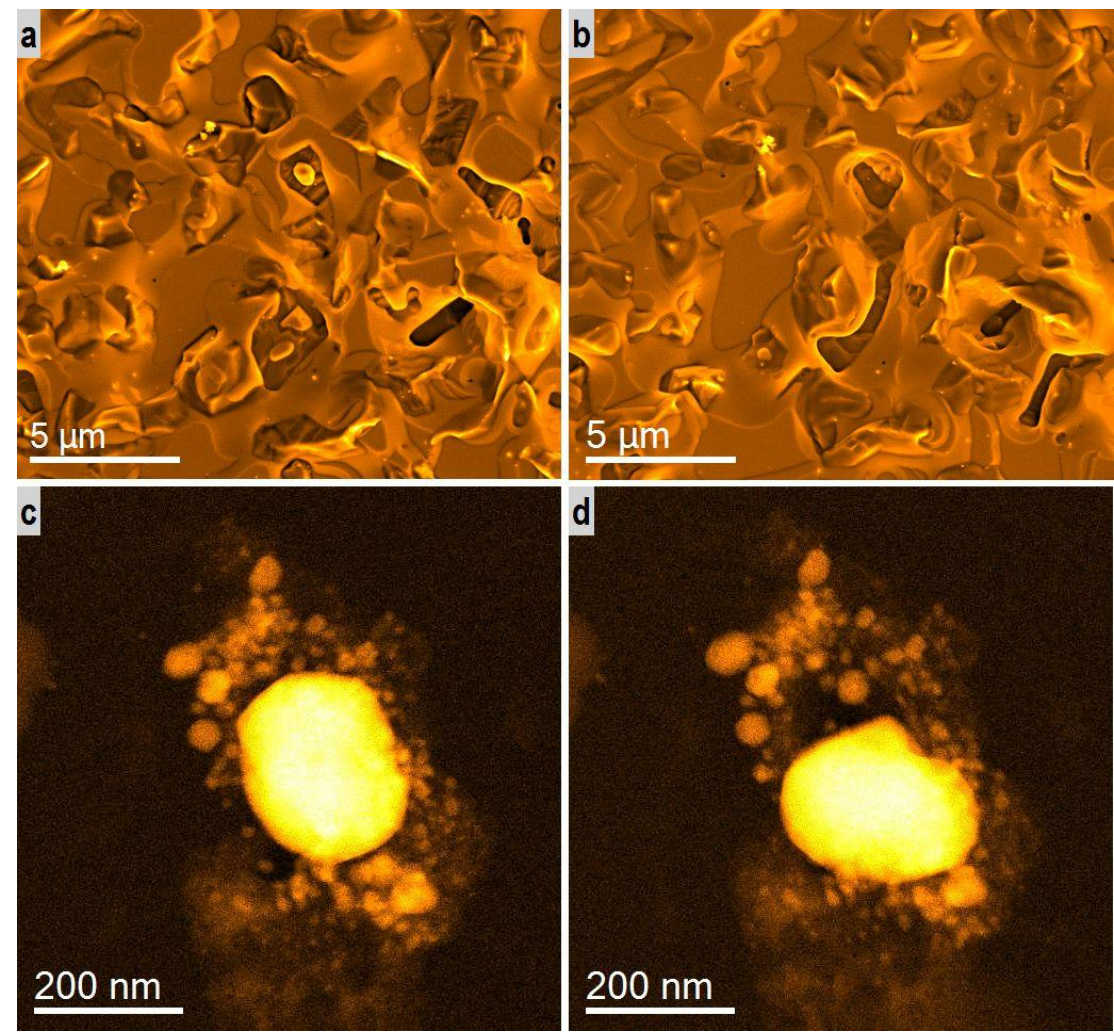

Figure 2. (a) and (b) show the aspect of a copper surface during hydrogen oxidation at 0.2 mbar and $650{ }^{\circ} \mathrm{C}$. Image (b) was recorded 8 minutes after (a) at the identical location using in situ SEM. STEM images (c) and (d) were recorded in situ and reveal dynamic shape changes of $\mathrm{Cu}$ nanoparticles caused by concurrent oxidation and reduction processes in an $\mathrm{H}_{2} / \mathrm{O}_{2}$ atmosphere. Image (d) was recorded $3 \mathrm{~s}$ after image (c). 